\title{
Are mobile device applications effective at supporting COPD self-management compared to usual care?
}

George Shaw, Maxine Whelan, Laura Armitage, Andrew Farmer

European Respiratory Journal 2019 54: PA747; DOI: 10.1183/13993003.congress-2019.PA747

\section{Abstract}

Background: Empowering people with chronic obstructive pulmonary disease (COPD) access using tools for self-monitoring of vital signs, behaviour and symptoms (including oxygen saturation, activity and chest tightness), or educational videos may support self-management. Currently it is unclear whether such tools are effective when delivered via mobile device applications.

Aim: Systematic review of randomised controlled trials (RCTs) to identify and report findings of trials using mobile applications.

Methods: MEDLINE, CINAHL, EMBASE and Cochrane Library were searched to July 2018 for published studies of RCTs that used mobile device applications (smartphone or tablet computerbased) in people with COPD, with or without healthcare provider input. Article screening and data extraction were completed by two authors. Risk of bias was also assessed.

Results: 1341 citations were retrieved and 12 RCTs were eligible for review. RCTs reported on quality of life $(n=12)$, number of exacerbations $(n=5)$, fatigue $(n=5)$, physical function $(n=5)$, dyspnoea $(n=4)$, physical activity $(n=5)$ and self-efficacy $(n=5)$. Statistically significant differences were reported for physical function (using the 6-minute walk test), quality of life (using the Short Form-12 and the functional component of the Clinical COPD Questionnaire) and activity (steps per day) compared with usual care ( $\mathrm{p}<0.05)$.

Conclusions: Use of mobile device applications by people with COPD may offer benefits in quality of life, physical function and activity levels; however, there was significant heterogeneity in the outcomes reported by the studies. Future trials should collect data on a standardised set of outcomes to allow comparison of findings. 